\title{
Dieulafoy's Lesion: Decade-Long Trends in Hospitalizations, Demographic Disparity, and Outcomes
}

Raja Chandra Chakinala ${ }^{1}$, Shantanu Solanki ${ }^{2}$, Khwaja F. Haq ${ }^{3}$, Jagmeet Singh ${ }^{4}$, Harshil Shah ${ }^{1}$, Dhanshree Solanki ${ }^{5}$, Asim Kichloo ${ }^{6}$, Khwaja S. Haq ${ }^{7}$, Azam H. Burney ${ }^{7}$, Shanza Waqar ${ }^{8}$, Manasee Vyas 9 , Savneek Chugh ${ }^{10}$, Christopher Nabors ${ }^{11}$

1. Internal Medicine, Independent Researcher, Sayre, USA 2. Hospital-Based Medicine, Geisinger Commonwealth School of Medicine, Scranton, USA 3. Gastroenterology, Henry Ford Hospital, Detroit, USA 4. Nephrology, Geisinger Commonwealth School of Medicine, Scranton, USA 5. Hospital Administration, Rutgers University, New Brunswick, USA 6. Internal Medicine, Central Michigan University, Saginaw, USA 7. Medicine, Kingsbrook Jewish Medical Center, Brooklyn, USA 8. Medicine, MedCare Clinics, St. Catharines, CAN 9. Medicine, Mahatma Gandhi Institute of Health Sciences, Mumbai, IND 10. Nephrology, Westchester Medical Center, Valhalla, USA 11. Internal Medicine, Westchester Medical Center, Valhalla, USA

Corresponding author: Raja Chandra Chakinala, rajachandra@gmail.com

\section{Abstract}

\section{Background}

Dieulafoy's lesion is a relatively rare, but potentially life-threatening, condition where a tortuous arteriole, most commonly in the stomach, may bleed and lead to significant gastrointestinal hemorrhage. Limited epidemiological data exist on patient characteristics and the annual number of hospitalizations associated with such lesions. The aim of our study is to determine the inpatient burden of Dieulafoy's lesion.

\section{Methods}

We analyzed the National Inpatient Sample (NIS) database for all subjects with a discharge diagnosis of Dieulafoy's lesion of the stomach, duodenum, and colon using International Classification of Diseases, 9th Revision, Clinical Modification (ICD-9-CM) codes 537.84 and 569.86 as the primary or secondary diagnosis during the period from 2002 to 2011. Statistical significance of variation in the number of hospital discharges and demographics during the study period was achieved using the Cochrane-Armitage trend test.

\section{Results}

In 2002, there were 1,071 admissions with a discharge diagnosis of Dieulafoy's lesion as compared to 7,414 in 2011 ( $p<0.0001$ ). Dieulafoy's lesion was found to be most common in the age group of 65-79 years $(p<$ $0.0001)$. Overall, it was found to be more common in males as compared to females $(p=0.0261)$. The white race was most commonly affected amongst all the races. The average cost of care per hospitalization

Received 06/30/2020 Review began $07 / 04 / 2020$ Review ended 07/05/2020 Published 07/13/2020

(c) Copyright 2020 Chakinala et al. This is an open access article distributed under the terms of the Creative Commons Attribution License CC-BY 4.0., which permits unrestricted use, distribution, and reproduction in any medium, provided the original author and source are credited. increased from $\$ 14,992$ in 2002 to $\$ 25,594$ in 2011 ( $\mathrm{p}<0.0001$ ).

\section{Conclusion}

There has been a steady rise in the number of inpatient admissions with Dieulafoy's lesions. Advances in diagnostic techniques likely play a key role in the higher detection rates along with the possible involvement of other unknown factors. Men, in the age group of 65 to 79 years, and Whites were found to have significantly higher admission rates than all other groups, with a significant increase in the cost of care.

Categories: Internal Medicine, Gastroenterology, Epidemiology/Public Health

Keywords: dieulafoy's lesion, gastrointestinal disorders, epidemiology, endoscopy, gastrointestinal bleeding, current trends

\section{Introduction}

Dieulafoy's lesion (DL) is a dilated submucosal artery that erodes the overlying intestinal mucosa in the absence of an underlying ulcer, aneurysm, or intrinsic mural abnormality [1]. DL is most commonly located in the stomach, but it is also reported to occur throughout the gastrointestinal (GI) tract [1]. It is underdiagnosed and accounts for $1 \%-2 \%$ of cases of acute GI bleeding [2]. Patients are typically asymptomatic until they present with GI bleeding, which may manifest as hematemesis, hematochezia, or melena. It's anatomy and conspicuous nature often pose a diagnostic challenge. Even after appropriate investigation, the lesion may remain obscure and cause a life-threatening hemorrhage, as the bleeding can be massive and recurrent [3].

The under-recognition contributes to its increased morbidity and previously reported high mortality 
associated with it. Given its impact on patient outcomes and healthcare costs, it is important to understand its epidemiology, patient demographics, associated comorbidities, and hospitalization trends. Currently, there are is no nationwide analysis highlighting the economic burden of DL in the US. The aim of our study is to determine the inpatient burden of DL in the US.

\section{Materials And Methods \\ Source of data}

The National Inpatient Sample (NIS), designed by the Agency for Healthcare Research and Quality (AHRQ), is the largest all-payer inpatient database in the U.S. Data are compiled yearly and contain discharge information from over 1200 hospitals located across 45 states in the U.S. The NIS was designed to approximate a $20 \%$ stratified sample of community hospitals in the country and provides sampling weights to calculate national estimates [4]. The NIS contains information included in a typical discharge summary, with safeguards in place to protect the privacy of individual patients, physicians, and hospitals. Each individual hospitalization is de-identified and maintained in the NIS as a unique entry, with one primary discharge diagnosis and approximately 24 secondary diagnoses during that hospitalization. Each entry also carries information on demographic details, insurance status, comorbidities, primary/secondary procedures, hospitalization outcomes, length of stay, and cost of care. The internal validity of the database is guaranteed by annual data quality assessments of the sample. Moreover, comparisons with data sources like the American Hospital Association (AHA) Annual Survey of Hospitals, National Hospital Discharge Survey from the National Center for Health Statistics, and MedPAR inpatient data from the Centers for Medicare and Medicaid Services strengthen the external validity of the sample [5-6].

\section{Study design}

This is a retrospective cohort study in which we queried the NIS database from the year 2002 to 2011 to identify all the hospitalizations with DL. We extracted data for all the hospitalizations from 2002 to 2011 with a primary or secondary diagnosis of DL, which, in turn, was identified with ICD-9 codes 537.84 (stomach and duodenum) and 569.86 (colon). Patients aged less than 18 years were excluded. Also, hospitalizations with missing information related to age, gender, admission/discharge date, in-hospital mortality status, demographics, and comorbidities were excluded as seen in previous well-designed studies [7]. To calculate the estimated cost of hospitalizations, NIS data were merged with the cost-to-charge ratio (CCR) files available from the Healthcare Cost and Utilization Project (HCUP). We estimated the cost of each inpatient stay by multiplying the total hospital charge with a cost-to-charge ratio.

\section{Variables and statistical analysis}

SAS 9.4 (SAS Institute Inc., Cary, North Carolina, USA) was utilized for complex statistical analyses. Since NIS represents a $20 \%$ stratified random sample of U.S. hospitals, analyses were performed using hospitallevel discharge weights provided by the NIS to obtain national estimates of hospitalizations. The frequency of paralytic ileus-related hospitalizations was calculated for each year. We calculated hospitalizations related to Dieulafoy's lesions per 1-million U.S. population by dividing yearly hospitalizations by $20 \%$ of the U.S. census population more than 18 years of age for that year [8]. The Cochrane-Armitage trend test was used to calculate trends in categorical variables [9]. The Wilcoxon rank-sum test was used to assess continuous variables. These hospitalizations were also calculated in subgroups of age (18-34, 35-49, 50-64, 65-79, and >80 years), gender, race (White, Black, Hispanic, and Others), insurance status (Medicare/Medicaid, private insurance, and self-pay/other), hospital location in different U.S. regions (Northeast, Midwest, South, and West), bed size of the hospital (small, medium, and large), and teaching status of the hospital (urban teaching, urban non-teaching, and rural). According to AHRQ, a hospital is considered to be a teaching hospital if it is: a) an AMA-approved residency program, b) a member of the Council of Teaching Hospitals or c) a hospital with a full-time resident-to-bed ratio more than 0.25 [10].

\section{Results}

\section{Demographics}

Patient characteristics are summarized in Table 1.

\begin{tabular}{|c|c|c|c|c|c|c|c|c|c|c|c|c|}
\hline Year & 2002 & 2003 & 2004 & 2005 & 2006 & 2007 & 2008 & 2009 & 2010 & 2011 & Average & P-VAL \\
\hline Number of obs. (n) & 1,071 & 4,972 & 5,175 & 4,940 & 5,303 & 5,417 & 6,231 & 6,380 & 6,685 & 7,414 & 5,359 & $<0.0001$ \\
\hline \multicolumn{13}{|l|}{ Age in years (\%) } \\
\hline 18-34 & 3.6 & 2.5 & 3.3 & 3.2 & 2.7 & 2.6 & 2.6 & 3.2 & 2.9 & 2.4 & 2.8 & 0.07 \\
\hline $35-49$ & 8.1 & 7.1 & 7.7 & 7.9 & 7.1 & 8.8 & 9.5 & 7.8 & 7.3 & 7.7 & 7.9 & 0.24 \\
\hline $50-64$ & 16.6 & 19.0 & 19.3 & 20.7 & 21.3 & 23.9 & 22.4 & 22.0 & 21.6 & 23.4 & 21.5 & $<0.0001$ \\
\hline
\end{tabular}




\section{Cureus}

\begin{tabular}{|c|c|c|c|c|c|c|c|c|c|c|c|c|}
\hline $65-79$ & 41.9 & 43.1 & 40.5 & 37.7 & 35.5 & 37.4 & 36.9 & 39.3 & 37.8 & 36.4 & 38.2 & $<0.0001$ \\
\hline$>=80$ & 29.4 & 28.0 & 29.1 & 30.1 & 33.2 & 27.3 & 28.3 & 27.6 & 30.2 & 29.8 & 29.3 & 0.4173 \\
\hline \multicolumn{13}{|l|}{ Gender (\%) } \\
\hline Male & 52.4 & 55.3 & 52.9 & 54.7 & 55.6 & 60.0 & 57.0 & 56.3 & 56.0 & 54.4 & 55.7 & 0.0261 \\
\hline Female & 47.6 & 44.7 & 47.0 & 45.2 & 44.4 & 40.0 & 43.1 & 43.7 & 44.0 & 45.6 & 44.2 & \\
\hline \multicolumn{13}{|l|}{ Race (\%) } \\
\hline White & 49.2 & 57.3 & 56.2 & 55.0 & 57.2 & 51.8 & 57.9 & 64.5 & 66.9 & 67.5 & 59.8 & 0.14 \\
\hline Black & 6.1 & 9.1 & 8.4 & 8.8 & 7.5 & 9.7 & 8.8 & 10.0 & 11.2 & 10.6 & 9.4 & 0.0748 \\
\hline Hispanic & 8.2 & 6.0 & 6.5 & 5.0 & 6.1 & 6.9 & 6.7 & 6.3 & 6.5 & 7.1 & 6.4 & 0.0043 \\
\hline Others & 4.9 & 3.3 & 3.9 & 3.2 & 3.8 & 4.4 & 5.3 & 3.9 & 5.5 & 5.2 & 4.4 & 0.0008 \\
\hline \multicolumn{13}{|l|}{ Region (\%) } \\
\hline Northeast & 17.6 & 19.7 & 20.4 & 21.9 & 23.1 & 20.3 & 20.4 & 20.0 & 20.1 & 20.3 & 20.6 & 0.2 \\
\hline Midwest & 23.1 & 22.8 & 20.7 & 21.8 & 24.0 & 24.6 & 23.3 & 22.8 & 22.1 & 23.6 & 22.9 & 0.0372 \\
\hline South & 38.0 & 38.9 & 39.5 & 36.7 & 32.3 & 32.5 & 36.0 & 39.0 & 39.2 & 37.8 & 37.0 & 0.1358 \\
\hline West & 21.3 & 18.6 & 19.4 & 19.7 & 20.7 & 22.7 & 20.3 & 18.1 & 18.6 & 18.4 & 19.6 & 0.0058 \\
\hline \multicolumn{13}{|l|}{ Location (\%) } \\
\hline Rural & 12.5 & 12.5 & 9.7 & 8.8 & 7.0 & 9.7 & 10.4 & 9.2 & 9.8 & 8.3 & 9.5 & $<0.0001$ \\
\hline Urban nonteaching & 48.2 & 46.8 & 46.8 & 50.2 & 44.1 & 44.9 & 44.5 & 42.9 & 41.2 & 39.3 & 44.2 & $<0.0001$ \\
\hline Urban teaching & 39.3 & 40.7 & 43.5 & 41.0 & 48.5 & 45.2 & 45.1 & 46.8 & 47.3 & 51.5 & 45.8 & $<0.0001$ \\
\hline \multicolumn{13}{|c|}{ Median household income (\%) } \\
\hline Quartile 1 & 3.2 & 22.9 & 22.8 & 22.8 & 24.2 & 25.2 & 24.9 & 23.5 & 26.3 & 26.4 & 24.1 & $<0.0001$ \\
\hline Quartile 2 & 19.9 & 24.6 & 29.1 & 24.4 & 23.4 & 23.5 & 27.5 & 26.6 & 24.0 & 23.9 & 25.1 & 0.15 \\
\hline Quartile 3 & 27.6 & 27.6 & 25.1 & 28.2 & 26.0 & 25.3 & 23.8 & 25.1 & 24.0 & 25.6 & 25.6 & $<0.0001$ \\
\hline Quartile 4 & 48.0 & 22.5 & 20.9 & 22.6 & 24.2 & 22.8 & 22.3 & 22.3 & 22.1 & 22.3 & 23.0 & $<0.0001$ \\
\hline \multicolumn{13}{|l|}{ Payment (\%) } \\
\hline Medicare & 69.2 & 69.6 & 68.7 & 68.8 & 68.2 & 64.1 & 63.8 & 66.7 & 65.6 & 67.1 & 66.9 & \\
\hline Medicaid & 4.4 & 4.6 & 4.4 & 5.6 & 4.8 & 6.3 & 6.0 & 6.3 & 7.9 & 6.6 & 5.9 & \\
\hline Private insurance & 20.7 & 19.4 & 20.7 & 20.0 & 20.7 & 23.0 & 23.1 & 19.7 & 20.1 & 20.8 & 20.8 & 0.16 \\
\hline Others (includes self-pay) & 5.7 & 6.2 & 6.1 & 5.7 & 6.3 & 6.6 & 7.1 & 7.3 & 6.2 & 5.1 & 6.3 & 0.38 \\
\hline \multicolumn{13}{|l|}{ Bed size } \\
\hline Small & 11.6 & 9.2 & 11.0 & 11.0 & 14.3 & 10.1 & 8.9 & 9.8 & 9.3 & 11.0 & 10.5 & 0.0373 \\
\hline Medium & 21.2 & 27.5 & 23.2 & 26.3 & 24.4 & 28.2 & 21.9 & 22.7 & 22.3 & 24.9 & 24.4 & 0.0003 \\
\hline Large & 67.2 & 63.4 & 65.8 & 62.7 & 61.0 & 61.5 & 69.3 & 66.5 & 66.7 & 63.2 & 64.7 & $<0.0001$ \\
\hline In-hospital mortality (\%) & 3.7 & 5.1 & 4.8 & 4.3 & 4.1 & 4.3 & 3.9 & 4.7 & 4.7 & 4.2 & 4.4 & $<0.0001$ \\
\hline Cost of care (\$) & 14,992 & 19,378 & 20,115 & 17,628 & 20,530 & 19,284 & 20,104 & 21,206 & 21,629 & 25,594 & 20,046 & $<0.0001$ \\
\hline AHRQ comorbidity measur & & & & & & & & & & & & \\
\hline
\end{tabular}




\section{Cureus}

\begin{tabular}{|c|c|c|c|c|c|c|c|c|c|c|c|c|}
\hline Obesity & 0.5 & 1.9 & 2.6 & 2.9 & 5.3 & 4.9 & 5.6 & 6.4 & 6.5 & 8.8 & 5.2 & $<0.0001$ \\
\hline Hypertension & 43.0 & 45.9 & 54.1 & 50.8 & 60.7 & 56.0 & 59.5 & 62.1 & 63.7 & 65.7 & 58.0 & $<0.0001$ \\
\hline Diabetes mellitus & 20.0 & 22.3 & 22.4 & 24.9 & 24.5 & 28.3 & 27.8 & 28.5 & 28.6 & 32.6 & 26.9 & $<0.0001$ \\
\hline Congestive heart failure & 17.3 & 18.4 & 15.4 & 21.0 & 21.3 & 19.9 & 17.9 & 19.4 & 22.3 & 21.2 & 19.7 & $<0.0001$ \\
\hline Chronic pulmonary disease & 20.0 & 16.9 & 19.1 & 19.8 & 24.0 & 19.9 & 18.9 & 20.2 & 23.2 & 22.2 & 20.6 & $<0.0001$ \\
\hline Peripheral vascular disease & 9.1 & 6.0 & 8.6 & 7.7 & 8.0 & 9.0 & 10.5 & 11.1 & 11.1 & 12.5 & 9.6 & $<0.0001$ \\
\hline Renal failure & 25.8 & 30.6 & 31.0 & 36.4 & 42.2 & 44.5 & 45.0 & 48.8 & 49.0 & 50.7 & 42.5 & $<0.0001$ \\
\hline Neurological disorders & 4.3 & 6.3 & 7.8 & 7.5 & 7.0 & 8.0 & 8.9 & 9.5 & 9.8 & 9.7 & 8.4 & $<0.0001$ \\
\hline Anemia & 24.7 & 23.5 & 25.4 & 24.1 & 28.1 & 28.9 & 28.9 & 31.6 & 32.9 & 32.8 & 71.2 & $<0.0001$ \\
\hline Solid tumor without metastasis & 7.5 & 2.4 & 1.7 & 2.2 & 1.7 & 2.5 & 1.7 & 2.4 & 2.1 & 3.1 & 2.3 & 0.43 \\
\hline Rheumatological disorders & 2.2 & 2.5 & 2.7 & 2.7 & 2.2 & 2.6 & 2.9 & 3.9 & 2.7 & 3.4 & 2.9 & $<0.0001$ \\
\hline Psychiatric disorders & 3.7 & 6.0 & 6.2 & 9.0 & 8.6 & 11.4 & 10.4 & 11.5 & 11.7 & 14.5 & 10.1 & $<0.0001$ \\
\hline Liver disease & 7.1 & 6.0 & 7.3 & 7.5 & 6.9 & 8.3 & 8.7 & 11.0 & 8.2 & 11.0 & 8.5 & $<0.0001$ \\
\hline
\end{tabular}

\section{TABLE 1: Baseline characteristics of Dieulafoy's lesion hospitalizations}

AHRQ: Agency for Healthcare Research and Quality

The number of hospitalizations with a discharge diagnosis of DL increased progressively from 1,071 admissions in 2002 as compared to 7,414 in 2011 ( $\mathrm{p}<0.0001$ ). A total of 53,588 hospitalizations with DL were reported during this time period. These patients were predominantly White (Figure 1 ) and in the 65 to 74 years age group (Figure 2). Men (55.7\%) accounted for a higher number of hospitalizations than women $(44.2 \%)$ and this ratio remained stable throughout the study period. The highest number of hospitalizations was seen in the South (37.0\%) and the lowest was seen in the West (19.6\%). Most hospitalizations were seen in urban teaching hospitals (45.8\%) followed by urban non-teaching hospitals (44.2\%) and rural hospitals (9.5\%). Hospitals with a large bed size accounted for $64.7 \%$ of the total hospitalizations. Medicare paid for $66.9 \%$ of the total hospitalizations whereas Medicaid paid for a mere $5.9 \%$ of the hospitalizations.

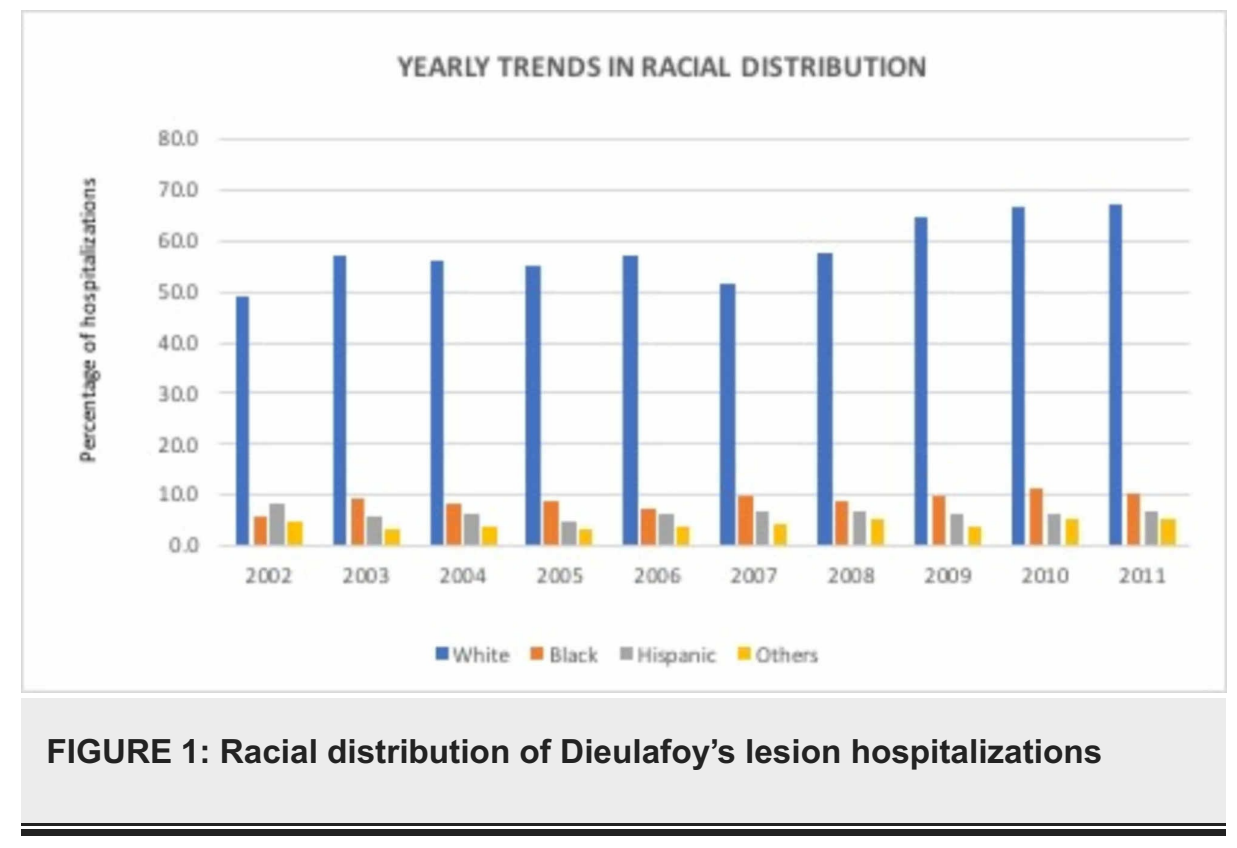




\section{Cureus}

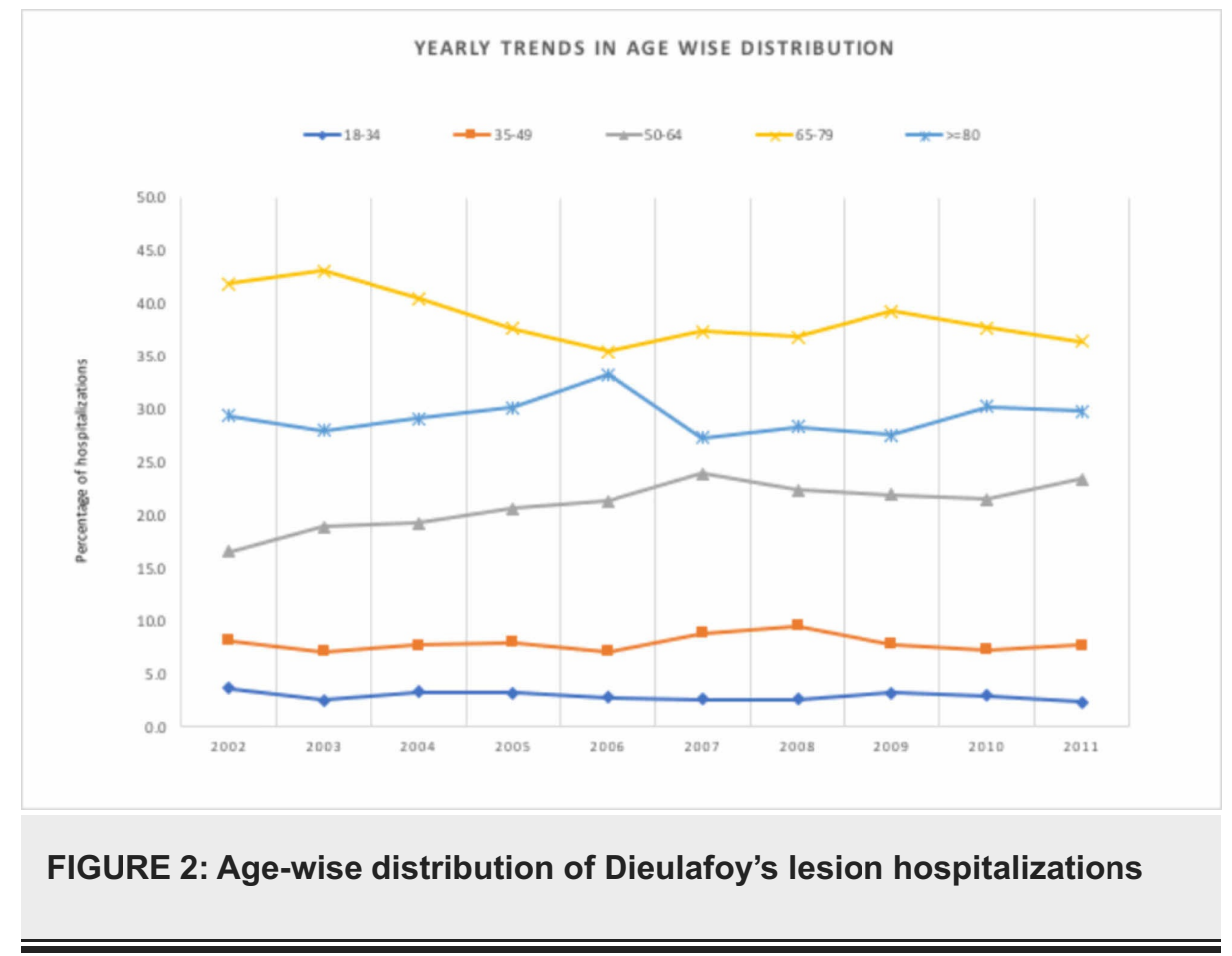

\section{Trends in hospitalizations}

The hospitalization rate increased significantly by $524 \%$ during the study period, from 25 to 156 per 1 million U.S. population per year from 2002 to 2011 ( $p<0.0001$; Table 2). The hospitalization rate increased significantly for the age group 50-64 ( $<0.0001$; Table 2$)$ and was higher in White men throughout the study period. The percent increase in hospitalizations was higher in men compared to women (550\% versus $499 \%$, $\mathrm{p}<0.0001$; Table 2) and for Blacks as compared with Whites (992\% versus $758 \%, \mathrm{p}<0.0001$; Table 2). The Northeast witnessed the highest percent increase in hospitalization and the West witnessed the lowest rate ( $622 \%$ vs $439 \%, p<0.0001$; Table 2 ). The respective mean ages (years) for the study sample were: overall 69.3 \pm SD 33.6 days, for men $67.7 \pm$ SD 33.8 days, and for women $71.5 \pm$ SD 32.6 days for women. The male-tofemale ratio differed for age groups $<80$ years versus $>=80$ years. In a sensitivity analysis, that ratio was $59.0 \%$ vs $40.9 \%$ in patients $<80$ years of age whereas it was $47.8 \%$ vs 52.3 in patients $>=80$ years of age (Figure $3)$.

\begin{tabular}{|c|c|c|c|c|c|c|c|c|c|c|c|c|c|}
\hline YEAR & 2002 & 2003 & 2004 & 2005 & 2006 & 2007 & 2008 & 2009 & 2010 & 2011 & Average & Percent Increase & P-value \\
\hline Per Million & 25 & 115 & 118 & 111 & 118 & 119 & 135 & 137 & 143 & 156 & 118 & 525.6 & $<0.0001$ \\
\hline \multicolumn{14}{|l|}{ Age in years } \\
\hline $18-34$ & 1 & 3 & 4 & 4 & 3 & 3 & 4 & 4 & 4 & 4 & 3 & 313.1 & $<0.0001$ \\
\hline $35-49$ & 2 & 8 & 9 & 9 & 8 & 11 & 13 & 11 & 10 & 12 & 9 & 495.3 & $<0.0001$ \\
\hline $50-64$ & 4 & 22 & 23 & 23 & 25 & 28 & 30 & 30 & 31 & 37 & 25 & 781.4 & $<0.0001$ \\
\hline 65-79 & 10 & 49 & 48 & 42 & 42 & 45 & 50 & 54 & 54 & 57 & 45 & 444.1 & $<0.0001$ \\
\hline$>=80$ & 7 & 32 & 34 & 34 & 39 & 33 & 38 & 38 & 43 & 46 & 34 & 534.0 & $<0.0001$ \\
\hline \multicolumn{14}{|l|}{ Gender } \\
\hline Male & 13 & 63 & 62 & 61 & 66 & 72 & 77 & 77 & 80 & 85 & 66 & 549.6 & $<0.0001$ \\
\hline Female & 12 & 51 & 55 & 50 & 52 & 48 & 58 & 60 & 63 & 71 & 52 & 499.1 & $<0.0001$ \\
\hline \multicolumn{14}{|l|}{ Race } \\
\hline White & 12 & 66 & 66 & 61 & 68 & 62 & 78 & 88 & 95 & 105 & 70 & 757.9 & $<0.0001$ \\
\hline Black & 2 & 10 & 10 & 10 & 9 & 12 & 12 & 14 & 16 & 17 & 11 & 992.4 & $<0.0001$ \\
\hline Hispanic & 2 & 7 & 8 & 6 & 7 & 8 & 9 & 9 & 9 & 11 & 8 & 438.4 & $<0.0001$ \\
\hline Others & 1 & 4 & 5 & 4 & 4 & 5 & 7 & 5 & 8 & 8 & 5 & 560.5 & $<0.0001$ \\
\hline
\end{tabular}




\section{Cureus}

\begin{tabular}{|c|c|c|c|c|c|c|c|c|c|c|c|c|c|}
\hline \multicolumn{14}{|l|}{ Region } \\
\hline Northeast & 4 & 23 & 24 & 24 & 27 & 24 & 28 & 27 & 29 & 32 & 24 & 622.0 & $<0.0001$ \\
\hline Midwest & 6 & 26 & 24 & 24 & 28 & 29 & 31 & 31 & 31 & 37 & 27 & 539.9 & $<0.0001$ \\
\hline South & 9 & 45 & 47 & 41 & 38 & 39 & 49 & 54 & 56 & 59 & 44 & 520.9 & $<0.0001$ \\
\hline West & 5 & 21 & 23 & 22 & 24 & 27 & 28 & 25 & 27 & 29 & 23 & 439.1 & $<0.0001$ \\
\hline \multicolumn{14}{|l|}{ Location } \\
\hline Rural & 3 & 14 & 11 & 10 & 8 & 12 & 14 & 13 & 14 & 13 & 11 & 315.6 & $<0.0001$ \\
\hline Urban nonteaching & 12 & 54 & 55 & 56 & 52 & 54 & 60 & 59 & 59 & 61 & 52 & 410.2 & $<0.0001$ \\
\hline Urban teaching & 10 & 47 & 51 & 46 & 57 & 54 & 61 & 64 & 67 & 80 & 54 & 720.0 & $<0.0001$ \\
\hline \multicolumn{14}{|l|}{ Median Household Income } \\
\hline Quartile 1 & 1 & 26 & 27 & 25 & 29 & 30 & 34 & 32 & 37 & 41 & 28 & 5122.0 & $<0.0001$ \\
\hline Quartile 2 & 5 & 28 & 34 & 27 & 28 & 28 & 37 & 36 & 34 & 37 & 30 & 649.5 & $<0.0001$ \\
\hline Quartile 3 & 7 & 32 & 30 & 31 & 31 & 30 & 32 & 34 & 34 & 40 & 30 & 480.7 & $<0.0001$ \\
\hline Quartile 4 & 12 & 26 & 25 & 25 & 29 & 27 & 30 & 31 & 31 & 35 & 27 & 191.2 & $<0.0001$ \\
\hline \multicolumn{14}{|l|}{ Payment } \\
\hline Medicare & 17 & 80 & 81 & 77 & 80 & 76 & 86 & 91 & 93 & 105 & 79 & 506.8 & $<0.0001$ \\
\hline Medicaid & 1 & 5 & 5 & 6 & 6 & 7 & 8 & 9 & 11 & 10 & 7 & 849.0 & $<0.0001$ \\
\hline Private insurance & 5 & 22 & 24 & 22 & 24 & 27 & 31 & 27 & 29 & 32 & 25 & 527.7 & $<0.0001$ \\
\hline Others (includes self-pay) & 1 & 7 & 7 & 6 & 7 & 8 & 10 & 10 & 9 & 8 & 7 & 458.0 & $<0.0001$ \\
\hline \multicolumn{14}{|l|}{ Bed size } \\
\hline Small & 3 & 11 & 13 & 12 & 17 & 12 & 12 & 13 & 13 & 17 & 12 & 495.0 & $<0.0001$ \\
\hline Medium & 5 & 31 & 27 & 29 & 29 & 34 & 30 & 31 & 32 & 39 & 29 & 634.5 & $<0.0001$ \\
\hline Large & 17 & 73 & 78 & 70 & 72 & 73 & 94 & 91 & 95 & 99 & 76 & 488.4 & $<0.0001$ \\
\hline
\end{tabular}

TABLE 2: Dieulafoy's lesion hospitalizations (\%) per hundred thousand hospitalizations

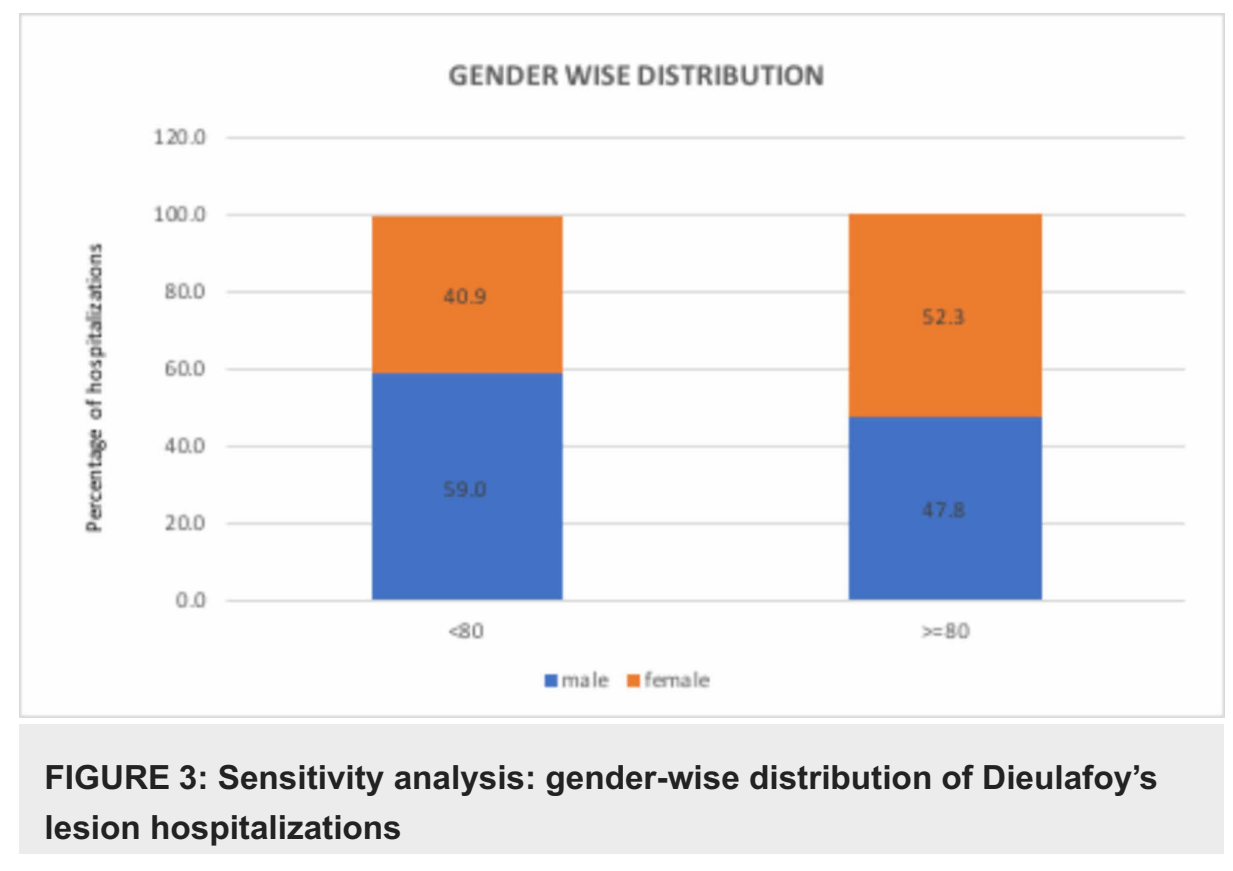




\section{Cureus}

\section{Length of stay and cost of care}

Median LOS was five days (interquartile range 3-9 days). There was a significant increase in the mean length of stay from 6.7 days to 8.7 days $(28.6 \%$ increase; $p=0.018$ ). The mean cost of care (adjusted for inflation) increased from $\$ 14,992$ per hospitalization in 2002 to $\$ 25,594$ in 2011 (70.7\% increase; $\mathrm{p}<0.0001$ ) (Table 1$)$. The total cost of all such hospitalizations increased from \$16 million in 2002 to $\$ 189$ million in 2011.

\section{AHRQ co-morbidities}

The most frequent coexisting conditions in these patients were anemia (71.2\%), hypertension (58.0\%), and renal failure (42.5\%) (Figure 4). The prevalence of several other comorbidities also increased significantly as depicted in Table 1 . It is very important to note obesity was identified as a comorbidity in $0.5 \%$ of the hospitalizations in 2002 whereas the percent contribution increased to $8.8 \%$ in 2011 accounting for a staggering 18 -fold increase.

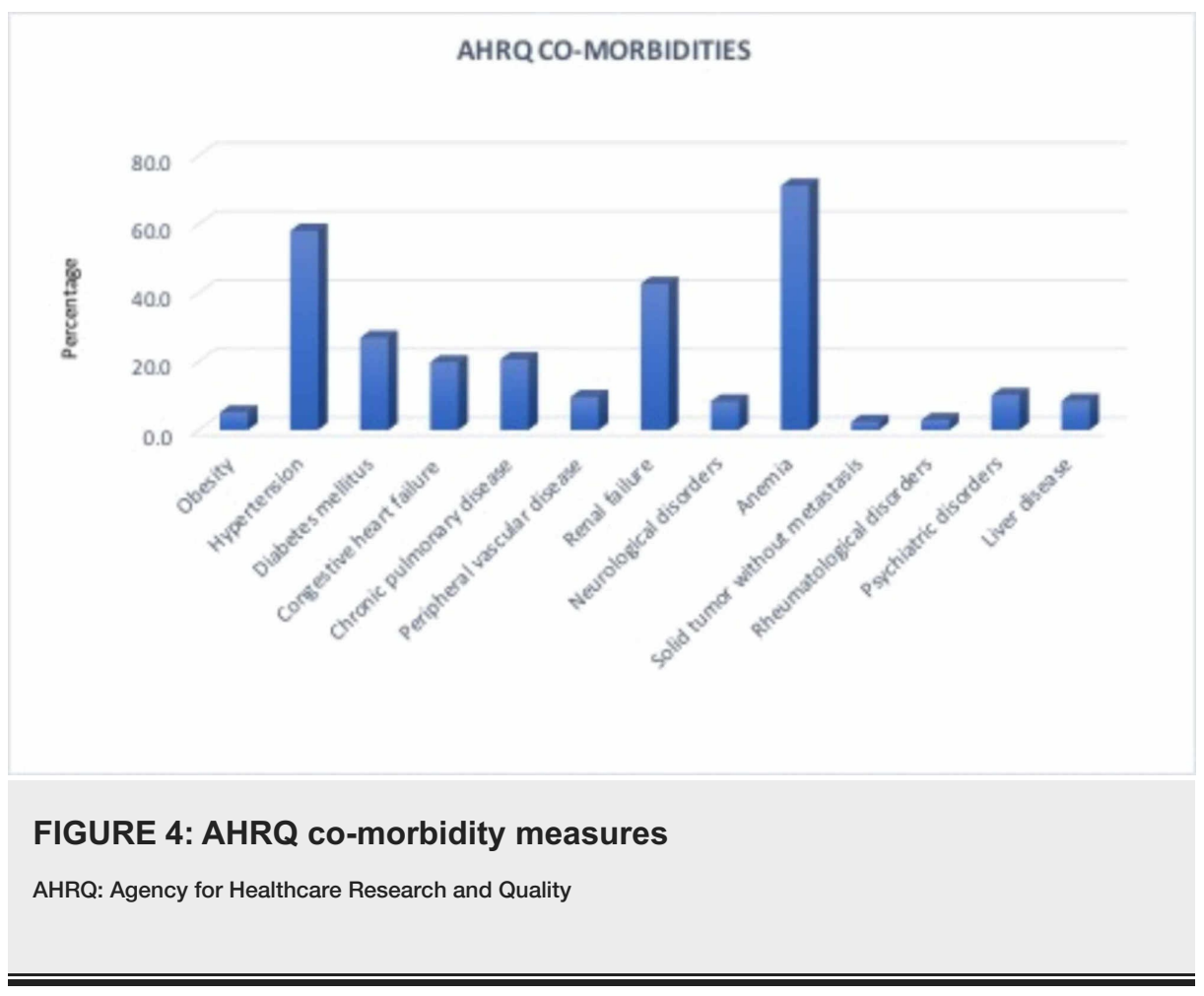

\section{All-cause inpatient mortality}

Overall in-hospital all-cause mortality associated with these hospitalizations was $4.4 \%$. It increased significantly from $3.7 \%$ in 2002 to $4.2 \%$ in 2011 (percent increase, $14.9 \%$; $<$ <0.0001) (Figure 5). The mortality rate was highest in the $\geqslant 80$-year age group at $5.2 \%$. The mortality rate was higher in males (4.8\%), Blacks (6.3\%), and in urban teaching hospitals (5.5\%) with a large bed size (4.7\%). Also, the mortality rate was noted to be highest in hospitalizations paid by Medicaid (7.1\%) (Table 3). 


\section{Cureus}

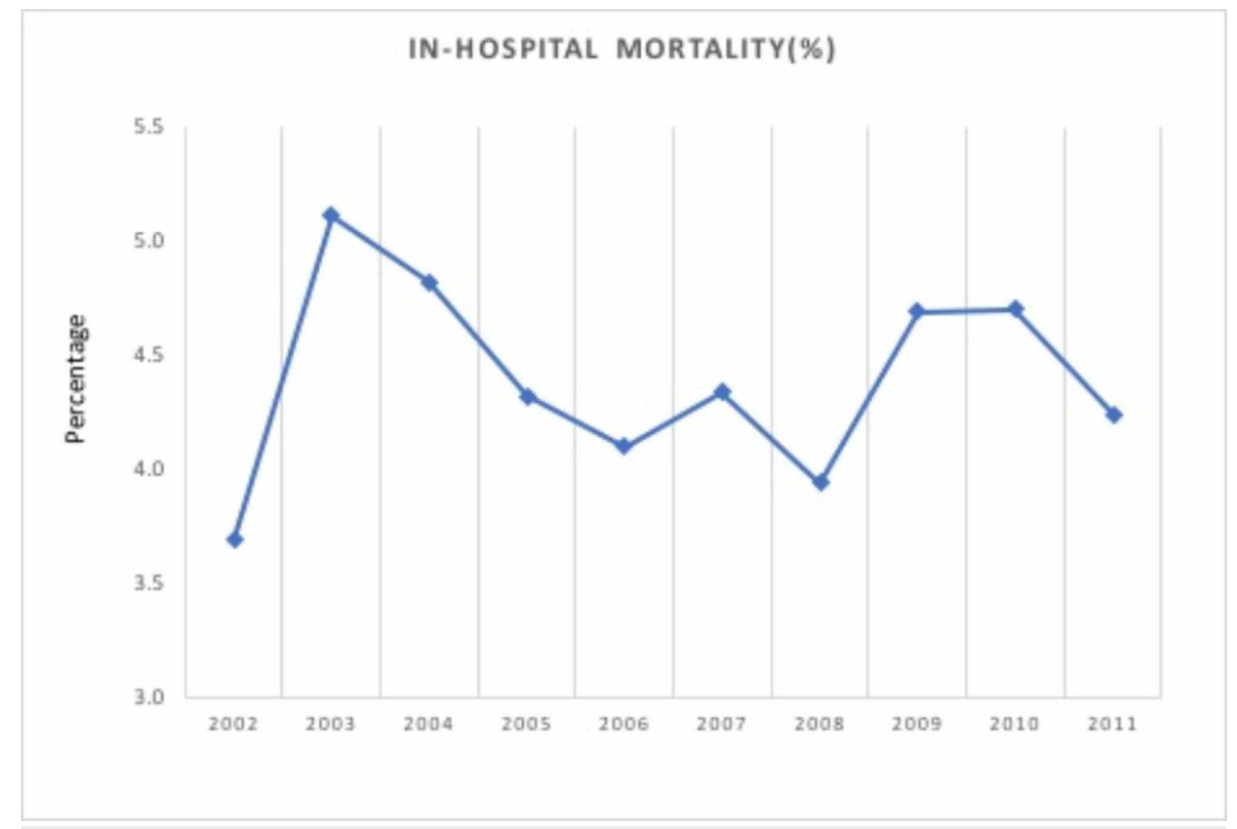

FIGURE 5: In-hospital mortality for Dieulafoy's lesion hospitalizations

\begin{tabular}{|c|c|c|c|c|c|c|c|c|c|c|c|c|c|}
\hline YEAR & 2002 & 2003 & 2004 & 2005 & 2006 & 2007 & 2008 & 2009 & 2010 & 2011 & Average & Percent Change & P-value \\
\hline Overall (\%) & 3.7 & 5.1 & 4.8 & 4.3 & 4.1 & 4.3 & 3.9 & 4.7 & 4.7 & 4.2 & 4.4 & 14.9 & $<0.0001$ \\
\hline \multicolumn{14}{|c|}{ Age in years (\%) } \\
\hline $18-34$ & 0.0 & 0.0 & 0.0 & 3.2 & 0.0 & 7.5 & 0.0 & 0.0 & 5.6 & 0.0 & 1.8 & * & 0.07 \\
\hline $35-49$ & 0.0 & 5.5 & 4.7 & 6.0 & 2.5 & 0.9 & 1.9 & 7.7 & 2.5 & 5.1 & 3.9 & * & 0.18 \\
\hline $50-64$ & 2.8 & 3.0 & 4.9 & 4.8 & 2.6 & 3.9 & 4.2 & 3.8 & 6.1 & 6.0 & 4.5 & 116.7 & 0.001 \\
\hline $65-79$ & 4.4 & 5.5 & 4.2 & 4.0 & 5.2 & 4.6 & 2.7 & 4.8 & 3.9 & 3.4 & 4.2 & -23.6 & 0.50 \\
\hline$>=80$ & 4.7 & 6.4 & 6.2 & 4.1 & 4.6 & 5.1 & 6.5 & 4.9 & 5.2 & 4.1 & 5.2 & -13.0 & 0.00 \\
\hline \multicolumn{14}{|l|}{ Gender (\%) } \\
\hline Male & 4.3 & 5.2 & 5.8 & 3.6 & 3.9 & 4.9 & 3.8 & 5.7 & 4.7 & 5.0 & 4.8 & 18.1 & 0.049 \\
\hline Female & 3.1 & 5.0 & 3.7 & 5.2 & 4.3 & 3.4 & 4.1 & 3.4 & 4.7 & 3.3 & 4.1 & 7.5 & \\
\hline \multicolumn{14}{|l|}{ Race (\%) } \\
\hline White & 3.8 & 4.6 & 5.4 & 3.5 & 3.6 & 4.2 & 4.0 & 5.1 & 4.5 & 4.2 & 4.4 & 11.1 & 0.08 \\
\hline Black & 7.9 & 8.0 & 4.2 & 6.8 & 6.3 & 5.3 & 6.7 & 5.4 & 8.1 & 5.4 & 6.3 & -31.4 & 0.04 \\
\hline Hispanic & 6.3 & 7.1 & 2.6 & 9.7 & 1.6 & 7.7 & 3.5 & 5.3 & 5.6 & 5.3 & 5.3 & -15.9 & 0.12 \\
\hline Others & 0.0 & 6.4 & 5.1 & 9.5 & 2.5 & 2.3 & 6.4 & 2.0 & 1.5 & 4.1 & 4.0 & * & 0.06 \\
\hline \multicolumn{14}{|l|}{ Region (\%) } \\
\hline Northeast & 5.53 & 3.4 & 6.3 & 4.9 & 5.5 & 4.2 & 4.0 & 5.6 & 5.3 & 5.1 & 5.0 & -7.4 & 0.12 \\
\hline Midwest & 6.1 & 4.3 & 5.8 & 4.1 & 4.1 & 3.6 & 2.4 & 5.8 & 3.5 & 2.6 & 4.0 & -56.7 & 0.0002 \\
\hline South & 1.2 & 4.0 & 3.3 & 4.5 & 3.8 & 4.8 & 4.9 & 3.5 & 5.7 & 4.5 & 4.3 & 278.3 & 0.11 \\
\hline West & 3.99 & 10.1 & 5.4 & 3.5 & 3.1 & 4.6 & 3.9 & 4.9 & 3.4 & 4.7 & 4.7 & 18.3 & 0.16 \\
\hline \multicolumn{14}{|l|}{ Location (\%) } \\
\hline Rural & 0.0 & 3.6 & 2.3 & 5.5 & 4.1 & 3.9 & 2.1 & 0.0 & 5.8 & 1.6 & 3.0 & * & 0.0089 \\
\hline
\end{tabular}




\section{Cureus}

\begin{tabular}{|c|c|c|c|c|c|c|c|c|c|c|c|c|c|}
\hline Urban nonteaching & 2.9 & 4.6 & 5.0 & 2.2 & 4.3 & 4.2 & 2.5 & 4.3 & 2.7 & 3.7 & 3.7 & 26.1 & $<0.0001$ \\
\hline Urban teaching & 5.8 & 6.2 & 5.2 & 6.6 & 3.9 & 4.6 & 5.8 & 6.1 & 6.2 & 4.9 & 5.5 & -15.5 & $<0.0001$ \\
\hline \multicolumn{14}{|c|}{ Median household income (\%) } \\
\hline Quartile 1 & 0.0 & 4.2 & 4.0 & 6.5 & 4.1 & 3.6 & 5.4 & 4.6 & 6.3 & 4.3 & 4.8 & * & 0.05 \\
\hline Quartile 2 & 2.3 & 6.5 & 4.4 & 4.0 & 4.7 & 6.7 & 4.2 & 4.4 & 4.8 & 4.0 & 4.7 & 72.5 & 0.05 \\
\hline Quartile 3 & 5.3 & 5.7 & 3.2 & 2.6 & 5.2 & 3.9 & 2.8 & 5.5 & 5.4 & 5.0 & 4.4 & -5.1 & 0.05 \\
\hline Quartile 4 & 3.7 & 3.9 & 8.2 & 4.6 & 2.8 & 3.3 & 3.5 & 4.0 & 1.7 & 3.5 & 3.8 & -5.4 & 0.05 \\
\hline \multicolumn{14}{|l|}{ Payment (\%) } \\
\hline Medicare & 4.0 & 6.2 & 4.8 & 4.0 & 5.1 & 4.7 & 4.4 & 5.0 & 4.4 & 3.7 & 4.6 & -9.2 & 0.11 \\
\hline Medicaid & 0.0 & 6.5 & 4.4 & 14.0 & 2.0 & 5.5 & 7.8 & 4.8 & 11.9 & 5.4 & 7.1 & * & 0.01 \\
\hline Private insurance & 4.4 & 2.1 & 2.7 & 3.1 & 2.2 & 4.0 & 1.3 & 3.1 & 3.2 & 5.9 & 3.2 & 35.2 & 0.0001 \\
\hline Others (includes self-pay) & 0.0 & 1.4 & 12.7 & 3.3 & 1.5 & 1.3 & 4.9 & 6.4 & 3.6 & 2.8 & 4.2 & * & 0.06 \\
\hline \multicolumn{14}{|l|}{ Bed size } \\
\hline Small & 0.0 & 2.3 & 3.7 & 5.2 & 3.0 & 4.9 & 4.5 & 2.6 & 2.8 & 3.9 & 3.6 & * & 0.002 \\
\hline Medium & 6.3 & 4.1 & 6.5 & 3.8 & 2.3 & 3.7 & 4.5 & 4.6 & 4.0 & 3.4 & 4.1 & -46.3 & $<0.0001$ \\
\hline Large & 3.5 & 6.0 & 4.4 & 4.4 & 5.1 & 4.6 & 3.7 & 5.1 & 5.2 & 4.5 & 4.7 & 27.9 & $<0.0001$ \\
\hline
\end{tabular}

\section{TABLE 3: All-cause in-hospital mortality (\%) for Dieulafoy's lesion hospitalizations}

*percentage change couldn't be calculated for groups with value "zero"

\section{Discussion}

To our knowledge, this is the first nationwide analysis of the inpatient burden of DL in the U.S. We found that the total number of hospitalizations with DL increased by nearly six-fold over the study period. It is currently unclear what factors are responsible for the increased prevalence of this condition among hospitalized patients. However, it is possible that several factors may be contributory. Medications like antiplatelet agents, non-steroidal anti-inflammatory drugs (NSAIDs), and anticoagulants, which could potentially cause rupture of submucosal vessels via erosive gastritis, are becoming more commonly used. And conditions such as chronic alcoholism (which may cause mucosal epithelial damage) and cardiovascular disease and renal failure (which may promote the formation of aberrant vessels that are prone to bleeding) are becoming increasingly common $[2,11-14]$. This may also partly be due to the increased awareness of DL during an endoscopic investigation of upper GI bleeding, resulting in a higher rate of detection.

Anemia, hypertension, and renal failure were found to be the most commonly associated co-morbidities. A similar association of DL with conditions that affect blood coagulation, such as chronic renal failure, cardiovascular disease, neurological, and liver disease, are well-described in the literature [14-18]. As previously suggested by the epidemiological data $[2,14-15,17]$, our analysis also showed the highest incidence per 1 million hospitalizations of DL in the elderly population, i.e. age groups $>50$ years with a mean age of 69.3 years. This association is not unexpected as the prevalence of cardiovascular, renal, and other comorbidities is known to increase with age.

Baxter et al. observed DL to be twice as common in males as compared to females [2]. Baettig et al. and Ibanez et al. reported a similar male predominance for DL in their retrospective studies [17-18]. However, Shin et al. reported the occurrence of DL in women two times as often as in men [11]. Our analysis like many others found DL to be more common in males (59\%) vs females (40.9\%) in the age group < 80 years, although the male-female ratio reversed to $47.8 \%$ vs $52.3 \%$ in the patients above 80 years of age. While we found higher incidence rates for inpatient DL hospitalization for Whites compared to other racial groups, the increase in hospitalization rate was higher in Blacks compared to Whites during the study period $(992.4 \%$ versus $757.9 \% ; \mathrm{p}<0.0001)$. The reasons for the difference in occurrence with gender and race is unclear and require further study.

The consequences of DL are significant, as patients are often at risk for re-bleeding, requiring multiple procedures, leading to other complications and prolonged hospital stay. Our findings show that the mean cost per hospitalization increased by $71 \%$ from 2002 to 2011 . The percent increase in the total annual cost of 
all such hospitalizations between 2002 to 2011 is $1081 \%$ ( $\$ 16$ million in 2002 to $\$ 189$ million in 2011). To our knowledge, this is the first study to report hospitalization costs in the US for DL.

In early reports, bleeding from DL was associated with high mortality $[2,16]$. Baxter et al. observed a decrease in mortality in DL patients from $80 \%$ to $8.3 \%$ [2]. Ibanez et al. reported a mortality rate of $4.9 \%$ during hospitalization for DL [17]. We found a mortality rate of $3.7 \%$ to $4.2 \%$ during the study period. Lower rates of mortality in contemporary studies may stem from a shift from a surgical approach to endoscopic management as the standard modality of management.

A strength of this study is the large sample size made possible through the use of the NIS database. Such a large sample size reduces bias inherent in studies that are confined to a single region or hospital. However, our analysis had a number of significant limitations. Administrative databases are susceptible to errors arising from coding inaccuracies. The diagnosis of DL and the presence of comorbidities were based on the presence of administrative codes. The database did not permit us to determine which hospitalizations assigned a diagnostic code of "DL" were hospitalized for the new diagnosis, as opposed to patients who had a diagnosis of DL in the past. Nor were we able to analyze the influence of medications on the prevalence of DL lesions.

There is a risk that our analysis could underestimate the number of hospitalizations with DL each year if an accompanying condition, such as "gastrointestinal bleeding," is listed as a primary diagnosis, even if a patient's primary diagnosis was DL. On the other hand, our analysis could also overestimate the number of patients with DL, as NIS considers each hospitalization as a separate entry, and there is no coding method that can separate index cases from readmissions.

Furthermore, the design of the database only allowed us to examine in-hospital characteristics. The study design limits the analysis of long-term follow-up outcomes or health care utilization in outpatient settings or emergency departments.

\section{Conclusions}

In conclusion, our review of hospitalization trends showed a significant increase in the number of DLrelated hospitalizations during the study period, along with an increase in the cost of care. The mortality rate associated with DL stayed low. Further studies are needed to identify factors contributing to the overall increase in the rates of DL and to permit the development of more effective diagnostic and treatment strategies for this entity.

\section{Additional Information \\ Disclosures}

Human subjects: Consent was obtained by all participants in this study. Animal subjects: All authors have confirmed that this study did not involve animal subjects or tissue. Conflicts of interest: In compliance with the ICMJE uniform disclosure form, all authors declare the following: Payment/services info: All authors have declared that no financial support was received from any organization for the submitted work. Financial relationships: All authors have declared that they have no financial relationships at present or within the previous three years with any organizations that might have an interest in the submitted work. Other relationships: All authors have declared that there are no other relationships or activities that could appear to have influenced the submitted work.

\section{References}

1. Nojkov B, Cappell MS: Gastrointestinal bleeding from Dieulafoy's lesion: clinical presentation, endoscopic findings, and endoscopic therapy. World J Gastrointest Endosc. 2015, 7:295-307. 10.4253/wjge.v7.i4.295

2. Baxter M, Aly EH: Dieulafoy's lesion: current trends in diagnosis and management. Ann R Coll Surg Engl 2010, 92:548-554. 10.1308/003588410X12699663905311

3. Chaer RA, Helton WS: Dieulafoy's disease. J Am Coll Surg. 2003, 196:290-296. 10.1016/S10727515(02)01801-X

4. Healthcare Cost and Utilization Project. Overview of the National (Nationwide) Inpatient Sample (NIS) . (2017). Accessed: January 17, 2018: https://www.hcup-us.ahrq.gov/nisoverview.jsp.

5. Healthcare Cost and Utilization Project. NIS related reports . (2012). Accessed: January 17, 2018: http://www.hcup-us.ahrq.gov/db/nation/nis/nisrelatedreports.jsp.

6. Badheka AO, Chothani A, Mehta K, et al.: Utilization and adverse outcomes of percutaneous left atrial appendage closure for stroke prevention in atrial fibrillation in the United States. Influence of hospital volume. Circ Arrhythm Electrophysiol. 2015, 8:42-48. 10.1161/CIRCEP.114.001413

7. Patel NJ, Deshmukh A, Pant S, et al.: Contemporary trends of hospitalization for atrial fibrillation in the United States, 2000 through 2010: implications for healthcare planning. Circulation. 2014, 129:2371-2379. 10.1161/CIRCULATIONAHA.114.008201

8. United States Census Bureau. Population and housing unit estimates . (2019). https://www.census.gov/programs-surveys/popest.html.

9. Armitage P: Tests for linear trends in proportions and frequencies . Biometrics. 1955, 11:375-386. $10.2307 / 3001775$ 


\section{Cureus}

10. Healthcare Cost and Utilization Project. HCUP methods series. (2002). Accessed: January 17, 2018: https://www.hcup-us.ahrq.gov/reports/methods/1998ChangesintheNISRedesignFinal.pdf.

11. Shin HJ, Ju JS, Kim KD, et al.: Risk factors for Dieulafoy lesions in the upper gastrointestinal tract . Clin Endosc. 2015, 48:228-233. 10.5946/ce.2015.48.3.228

12. Lee YT, Walmsley RS, Leong RW, Sung JJ: Dieulafoy's lesion. Gastrointest Endosc. 2003, 58:236-43. 10.1067/mge.2003.328

13. Chung IK, Kim EJ, Lee MS, et al.: Bleeding Dieulafoy's lesions and the choice of endoscopic method: comparing the hemostatic efficacy of mechanical and injection methods. Gastrointest Endosc. 2000, 52:721724. 10.1067/mge.2000.108040

14. Ding YJ, Zhao L, Liu J, Luo HS: Clinical and endoscopic analysis of gastric Dieulafoy's lesion. World J Gastroenterol. 2010, 16:631-635. 10.3748/wjg.v16.i5.631

15. Marchese M, De Cristofaro R, Federici AB, et al.: Duodenal and gastric Dieulafoy's lesions in a patient with type 2A von Willebrand's disease. Gastrointest Endosc. 2005, 61:322-325. 10.1016/S0016-5107(04)02636-7

16. Schmulewitz N, Baillie J: Dieulafoy lesions: a review of 6 years of experience at a tertiary referral center. Am J Gastroenterol. 2001, 96:1688-1694.

17. Ibáñez A, Castro E, Fernández E, et al.: Clinical aspects and endoscopic management of gastrointestinal bleeding from Dieulafoy's lesion [Article in Spanish]. Rev Esp Enferm Dig. 2007, 99:505-510. 10.4321/s113001082007000900005

18. Baettig B, Haecki W, Lammer F, Jost R: Dieulafoy's disease: endoscopic treatment and follow up . Gut. 1993, 34:1418-1421. 10.1136/gut.34.10.1418 